\title{
On a nonstationary nonlinear coupled system*
}

\author{
GANG $\mathrm{LI}^{1}$, HUI WANG ${ }^{1}$ and JIANG $\mathrm{ZHU}^{2}$ \\ ${ }^{1}$ Department of Mathematics, Nanjing University of Information Science \\ \& Technology, 210044 Nanjing, China \\ ${ }^{2}$ Laboratório Nacional de Computação Científica, MCT, \\ Avenida Getúlio Vargas, 333, 25651-075 Petrópolis, RJ, Brazil \\ E-mails: ligang@nuist.edu.cn / huispecial@gmail.com / jiang@lncc.br
}

\begin{abstract}
In this paper, a strongly nonlinear coupled elliptic-parabolic system modelling a class of engineering problems with heat effect is studied. Existence of a weak solution is first established by Schauder fixed point theorem, where the coupled functions $\sigma(s), k(s)$ are assumed to be bounded. The uniqueness of the solution is obtained by applying Meyers' theorem and assuming that $\sigma(s), k(s)$ are Lipschitz continuous. The regularity of the solution is then analyzed in dimension $d \leq 2$ under the assumptions on $\sigma(s), k(s) \in C^{2}(\mathbb{R})$ and the boundedness of their derivatives of second order. Finally, the blow-up phenomena of the system are studied.
\end{abstract}

Mathematical subject classification: $35 \mathrm{~J} 60,35 \mathrm{~K} 55$.

Key words: elliptic-parabolic system, existence, uniqueness, regularity, blow-up.

\section{Introduction}

In many engineering problems, cf. $[1,2,3]$, and the references therein, we encounter an incompressible quasi Newtonian flows with viscous heating which

\#CAM-225/10. Received: 18/VI/10. Accepted: 18/IX/10.

*This work was supported partially by the National Natural Science Foundation of China (40537034) and the Brazilian National Council for Scientific and Technological Development (CNPq). 
can be modelled as:

$$
\begin{cases}\text { (i) } & -2 \nabla \cdot(\sigma(\theta) D(\boldsymbol{u}))+\nabla p=\boldsymbol{f} \\ \text { (ii) } & \nabla \cdot \boldsymbol{u}=0 \\ \text { (iii) } & \theta_{t}-\nabla \cdot(k(\theta) \nabla \theta)+\boldsymbol{u} \cdot \nabla \theta+c \theta=\sigma(\theta)|D(\boldsymbol{u})|^{2}\end{cases}
$$

where $\boldsymbol{u}$ is the velocity, $p$ the pressure, $\theta$ the temperature. The viscosity $\sigma$ is a function of $\theta$,

$$
D(\boldsymbol{u})=\frac{1}{2}\left(\nabla \boldsymbol{u}+\nabla \boldsymbol{u}^{T}\right)
$$

is the strain rate tensor, and $|D(\boldsymbol{u})|^{2}$ is the second invariant of $D(\boldsymbol{u})$.

Problems of this type have received especial attention recently, cf. $[2,4,5]$. Very similar problems can be found in modelling turbulent flows, cf. [6, 7], thermistor problems, cf. [9, 10, 12, 15, 13, 14, 16, 19, 23, 22, 24, 26, 29, 31, 32], semiconductor devices, cf. [30, 17, 28], electromagnetic "induction heating" problems, cf. [11], and so on. The main difficulties in analysis of the system (1) come from the strongly coupled nonlinearity and the incompressibility (from numerical point of view). In this paper, we focus the first difficulty and consider its simplified scalar model:

$$
\left\{\begin{array}{lll}
\text { (i) } \quad-\nabla \cdot(\sigma(\theta) \nabla u)=f & \text { in } \Omega \times(0, T), \\
\text { (ii) } \quad \theta_{t}-\nabla \cdot(k(\theta) \nabla \theta)+\boldsymbol{b} \cdot \nabla \theta+c \theta=\sigma(\theta)|\nabla u|^{2} & \text { in } \Omega \times(0, T), \\
\text { (iii) } \quad u=0, \quad \theta=0 & \text { on } \Gamma \times(0, T), \\
\text { (iv) } \quad \theta(x, 0)=\theta_{0}(x) & \text { in } \Omega .
\end{array}\right.
$$

where $u, \theta: \Omega \times(0, T) \rightarrow \mathbb{R}$ are unknowns, $\Omega$ is a bounded open subset of $\mathbb{R}^{d}, d=1,2$ or $3, \Gamma$ its regular boundary, $T$ is some positive given number. $\boldsymbol{b}, c$ are given vector and scalar functions. We will study the problem (1) in next work.

The model problem (2) can be also thought, from mathematical point of view, as a generalization of thermistor problem where $u$ is potential, $\theta$ temperature and $f, \boldsymbol{b}, c \equiv 0$. It is necessary and important to understand well such a fundamental model problem (2) in simulating quasi Newtonian flows with viscous heating, turbulent flows, thermistor problems, semiconductor devices, electromagnetic "induction heating" problems, etc. 
Following the works by Antontsev-Chipot [10] and Elliott-Larsson [16] for thermistor problem, we give in this paper a complete analysis such as existence, uniqueness, regularity and blow-up of the problem (2). While in [24] the authors assumed $f \in L^{2}(\Omega)$ to get the existence of the solution, we establish similar results with a weaker assumption on it (see Theorem 1 below), which also extends results in $[10,16]$, where simply $f=0$ is involved in the partial differential equation. By applying Meyers' estimate from [21], regularity assumptions on the solution such that

$$
\nabla u_{i}, \nabla \phi_{2} \in L^{2 q /(q-n)}\left(0, T ; L^{q}(\Omega)\right), i=1,2
$$

and

$$
\nabla \phi_{1} \in L^{4 q /(q-n)}\left(0, T ; L^{q}(\Omega)\right), q>\max (n, 2)
$$

etc. in [10] are not needed to reach the uniqueness of the solution. And a non-trivial extension of the blow-up analysis in [10] to the case of diffusionconvection-reaction is presented following the idea from [8].

The paper is constructed as follows. In section 2 we formulate the variational form of the problem. And the following two sections are devoted to analyze the existence and uniqueness of the weak solution. Then we study the regularity of the solution. Finally, we discuss the blow-up.

\section{Variational formulation}

We will use standard notation for the spaces and corresponding norms. Let $W^{m, s}(\Omega)$ denotes the standard sobolev space, with its norm $\|\cdot\|_{W^{m, s}(\Omega)}$, for $m \geq 0$ and $1 \leq s \leq \infty$. We write $H^{m}(\Omega)=W^{m, s}(\Omega)$ when $s=2$, with the norm $\|\cdot\|_{W^{m, 2}(\Omega)}$, and $L^{s}(\Omega)=W^{0, s}(\Omega)$ when $m=0$, with the norm $\|\cdot\|_{L^{s}(\Omega)}$. $W_{0}^{m, s}(\Omega)$ is the closure of the space $C_{0}^{\infty}(\Omega)$ for the norm $\|\cdot\|_{W^{m, s}(\Omega)}$. When considering space-time functions $v(x, t),(x, t) \in \Omega \times(0, T)$, we define the space $L^{r}(0, T ; X)(1 \leq r<\infty)$ (where $X$ is a Sobolev space on $\Omega$ ) as:

$$
L^{r}(0, T ; X):=\left\{v:(0, T) \rightarrow X \mid \int_{0}^{T}\|v(t)\|_{X}^{r} d t<\infty\right\} .
$$

In a similar way we can define $L^{\infty}(0, T ; X)$ and $C(0, T ; X)$. Vector variables are, in general, denoted with bold face. 
We assume that $\theta_{0} \in L^{2}(\Omega)$, and let $V=H_{0}^{1}(\Omega), V^{\prime}=H^{-1}(\Omega)$ the dual space of $V$. Then, for a given $f \in L^{\infty}\left(0, T ; V^{\prime}\right)$, the variational formulation of the problem (2) can be defined as:

$$
\begin{cases}\text { Find } u \in L^{\infty}(0, T ; V), \theta \in L^{2}(0, T ; V) \cap C\left([0, T] ; L^{2}(\Omega)\right), \\ \quad \theta_{t} \in L^{2}\left(0, T ; V^{\prime}\right) \text { such that } & \forall v \in V \\ \text { (i) } \quad a(\theta ; u, v)=(f, v), & \forall \eta \in V \cap L^{\infty}(\Omega) \\ \text { (ii) } \quad\left(\theta_{t}, \eta\right)+b(\theta ; \theta, \eta)=\left(\sigma(\theta)|\nabla u|^{2}, \eta\right), & \forall \eta \in\end{cases}
$$

where

$$
\begin{gathered}
a(\theta ; u, v)=(\sigma(\theta) \nabla u, \nabla v), \\
b(\xi ; \theta, \eta)=(k(\xi) \nabla \theta, \nabla \eta)+(\boldsymbol{b} \cdot \nabla \theta, \eta)+(c \theta, \eta),
\end{gathered}
$$

and $(\cdot, \cdot)$ denotes the inner product of $\left[L^{2}(\Omega)\right]^{d}$ or the duality between $\left[L^{s}(\Omega)\right]^{d}$ and $\left[L^{s^{\prime}}(\Omega)\right]^{d}, s^{\prime}$ is the dual number of $s$.

\section{Existence of a weak solution}

We assume that $\sigma, k \in C(\mathbb{R})$ satisfying

$$
0<\sigma_{1} \leq \sigma(\theta) \leq \sigma_{2}<\infty ; \quad 0<k_{1} \leq k(\theta) \leq k_{2}<\infty
$$

where $\sigma_{i}, k_{i}$ are positive constants. $C$ denotes a generic constant depending on $\Omega, d$ and $\sigma_{i}, k_{i}$.

It is easy to see that, for any given $\theta$, and $v, w \in V$

$$
\begin{gathered}
a(\theta ; v, v) \geq \sigma_{1}\|\nabla v\|_{L^{2}(\Omega)}^{2}, \\
|a(\theta ; v, w)| \leq \sigma_{2}\|\nabla v\|_{L^{2}(\Omega)}\|\nabla w\|_{L^{2}(\Omega)}
\end{gathered}
$$

where $\|\nabla \cdot\|_{L^{2}}$ is equivalent to the norm $\|\cdot\|_{V}$ by Poincaré inequality (cf. p. 11 in [25]).

To symmetrize the trial and test function spaces of (3.ii), we note that in [24, 32, 33] Meyer's estimate was applied and some regularity assumption in 3D case was needed. Here we are doing in a different way which is based on the Maximum principle and that regularity assumption is then unnecessary. 
Lemma 1. For any given $\theta$, the solution to (3.i) $u$ satisfies that

$$
\|\nabla u\|_{L^{\infty}\left(0, T ; L^{2}(\Omega)\right)} \leq C\|f\|_{L^{\infty}\left(0, T ; V^{\prime}\right)} .
$$

Moreover, if $f \in L^{2}\left(0, T ; L^{q}(\Omega)\right)$ where $q>d / 2$, then

$$
\|u\|_{L^{2}\left(0, T ; L^{\infty}(\Omega)\right)} \leq C\|f\|_{L^{2}\left(0, T ; L^{q}(\Omega)\right)} .
$$

Proof. Let $v=u$ in (3.i), and notice that (7), we can get (9). (10) is a consequence of Theorem 8.16 in [20].

By (2.i), the right-hand side of (2.ii) can be written as

$$
\sigma(\theta)|\nabla u|^{2}=\nabla \cdot(\sigma(\theta) u \nabla u)+f u .
$$

Then, for any $\eta \in V$, we have

$$
\begin{aligned}
\left(\sigma(\theta)|\nabla u|^{2}, \eta\right) & =-(\nabla \cdot(\sigma(\theta) u \nabla u)+f u, \eta) \\
& =(\sigma(\theta) u \nabla u, \nabla \eta)+(f u, \eta) .
\end{aligned}
$$

By Lemma 1 , if $f \in L^{\infty}\left(0, T ; V^{\prime}\right) \cap L^{2}\left(0, T ; L^{q}(\Omega)\right)$, then (12) defines an element of $L^{2}\left(0, T ; V^{\prime}\right)$. Thus, we can rewrite (3) equivalently as:

Find $u \in L^{\infty}(0, T ; V), \theta \in L^{2}(0, T ; V) \cap C\left([0, T] ; L^{2}(\Omega)\right)$,

$$
\theta_{t} \in L^{2}\left(0, T ; V^{\prime}\right) \text { such that }
$$

(i) $a(\theta ; u, v)=(f, v), \quad \forall v \in V$

(ii) $\left(\theta_{t}, \eta\right)+b(\theta ; \theta, \eta)=\left(\sigma(\theta)|\nabla u|^{2}, \eta\right), \quad \forall \eta \in V$

Problem (13) is easier to study since its trial and test function spaces are same.

Since

$$
(\boldsymbol{b} \cdot \nabla \theta, \theta)=-(\nabla \cdot(\boldsymbol{b} \theta), \theta)=-(\boldsymbol{b} \cdot \nabla \theta, \theta)-(\nabla \cdot \boldsymbol{b} \theta, \theta)
$$

or

$$
(\boldsymbol{b} \cdot \nabla \theta, \theta)=-\frac{1}{2}(\nabla \cdot \boldsymbol{b} \theta, \theta) .
$$

So, if

$$
c-\frac{1}{2} \nabla \cdot \boldsymbol{b} \geq-\left(k_{1}-p\right) \lambda_{\min },
$$


where $\lambda_{\text {min }}$ denotes the smallest eigenvalue of $-\Delta$ in $\Omega, p>0$ and if

$$
\boldsymbol{b} \in\left[L^{3}(\Omega)\right]^{d}, c \in L^{3 / 2}(\Omega),
$$

we have, for any $\xi, \theta, \eta \in V$

$$
\begin{aligned}
b(\xi ; \eta, \eta) \geq & k_{1}\|\nabla \eta\|_{L^{2}(\Omega)}^{2}+\left(\left[c-\frac{1}{2} \nabla \cdot \boldsymbol{b}\right] \eta, \eta\right) \geq \alpha\|\nabla \eta\|_{L^{2}(\Omega)}^{2}, \\
|b(\xi ; \theta, \eta)| \leq & k_{2}\|\nabla \theta\|_{L^{2}(\Omega)}\|\nabla \eta\|_{L^{2}(\Omega)}+\|\boldsymbol{b}\|_{L^{3}(\Omega)}\|\nabla \theta\|_{L^{2}(\Omega)}\|\eta\|_{L^{6}(\Omega)} \\
& +\|c\|_{L^{3 / 2}(\Omega)}\|\theta\|_{L^{6}(\Omega)}\|\eta\|_{L^{6}(\Omega)} \\
\leq & \beta\|\nabla \theta\|_{L^{2}(\Omega)}\|\nabla \eta\|_{L^{2}(\Omega)}
\end{aligned}
$$

where $\alpha=\min \left(k_{1}, p\right), \beta=\beta\left(k_{2}, \boldsymbol{b}, c\right)$ is a constant.

Then we can prove the following:

Theorem 1. (Existence) If $f \in L^{\infty}\left(0, T ; V^{\prime}\right) \cap L^{2}\left(0, T ; L^{q}(\Omega)\right)(q>d / 2)$, and $\boldsymbol{b}, c$ functions satisfying (15), then there exists a weak solution $\{u, \theta\}$ to problem (13) such that

$$
\begin{aligned}
& \|\nabla u\|_{L^{\infty}\left(0, T ; L^{2}(\Omega)\right)} \leq C\|f\|_{L^{\infty}\left(0, T ; V^{\prime}\right)}, \\
& \|u\|_{L^{2}\left(0, T ; L^{\infty}(\Omega)\right)} \leq C\|f\|_{L^{2}\left(0, T ; L^{q}(\Omega)\right)}, \\
& \|\theta\|_{C\left(0, T ; L^{2}(\Omega)\right)}^{2}+\|\theta\|_{L^{2}(0, T ; V)}^{2} \\
\leq & C\left(\|f\|_{L^{\infty}\left(0, T ; V^{\prime}\right)}^{2}\|f\|_{L^{2}\left(0, T ; L^{q}(\Omega)\right)}^{2}+\left\|\theta_{0}\right\|_{L^{2}(\Omega)}^{2}\right) .
\end{aligned}
$$

Proof. Choose $\xi \in L^{2}\left(0, T ; L^{2}(\Omega)\right)$, since (7), (8) hold, we denote by $u_{\xi} \in V$ the solution of

$$
a\left(\xi ; u_{\xi}, v\right)=(f, v), \quad \forall v \in V
$$

in view of the Lax-Milgram Theorem.

According the Theorem 2.1 in [10], there exists a unique $\theta_{\xi} \in L^{2}(0, T ; V) \cap$ $C\left(0, T ; L^{2}(\Omega)\right)$ with $\theta_{\xi_{t}} \in L^{2}\left(0, T ; V^{\prime}\right)$ and $\theta_{\xi_{0}}=\theta_{0}$ the solution of

$$
\begin{aligned}
& \left(\theta_{\xi_{t}}, \eta\right)+\left(k(\xi) \nabla \theta_{\xi}, \nabla \eta\right)+\left(\boldsymbol{b} \cdot \nabla \theta_{\xi}, \eta\right)+\left(c \theta_{\xi}, \eta\right) \\
& \quad=-\left(\sigma(\xi) u_{\xi} \nabla u_{\xi}, \nabla \eta\right)+\left(f u_{\xi}, \eta\right), \quad \forall \eta \in V
\end{aligned}
$$


together with $k=k(\xi)$. Let us consider the map

$$
\xi \rightarrow \theta_{\xi}=J(\xi) .
$$

This map carries $L^{2}\left(0, T ; L^{2}(\Omega)\right)$ into itself. Moreover, since (15) holds, by (6) and (17), if choosing $\eta=\theta_{\xi}$ in (23) and integrating in $t$, we have

$$
\begin{aligned}
& \frac{1}{2}\left\|\theta_{\xi}\right\|_{L^{2}(\Omega)}^{2}+\alpha \int_{0}^{t}\left\|\nabla \theta_{\xi}\right\|_{L^{2}(\Omega)}^{2} d t \\
\leq & \frac{1}{2}\left\|\theta_{\xi}\right\|_{L^{2}(\Omega)}^{2}+\int_{0}^{t}\left[\left(k(\xi) \nabla \theta_{\xi}, \nabla \theta_{\xi}\right)+\left(\boldsymbol{b} \cdot \nabla \theta_{\xi}, \theta_{\xi}\right)+\left(c \theta_{\xi}, \theta_{\xi}\right)\right] d t \\
\leq & \left|\int_{0}^{t}\left(\sigma(\xi) u_{\xi} \nabla u_{\xi}, \nabla \theta_{\xi}\right) d t\right|+\left|\int_{0}^{t}\left(f u_{\xi}, \theta_{\xi}\right) d t\right|+\frac{1}{2}\left\|\theta_{0}\right\|_{L^{2}(\Omega)}^{2} .
\end{aligned}
$$

By Hölder, Young's inequalities, the first two terms of the right-hand side of the last equation follows,

$$
\begin{aligned}
& \left|\int_{0}^{t}\left(\sigma(\xi) u_{\xi} \nabla u_{\xi}, \nabla \theta_{\xi}\right) d t\right| \\
\leq & \sigma_{2} \int_{0}^{t}\left\|u_{\xi}\right\|_{L^{\infty}(\Omega)}\left\|\nabla u_{\xi}\right\|_{L^{2}(\Omega)} \cdot\left\|\nabla \theta_{\xi}\right\|_{L^{2}(\Omega)} d t \\
\leq & \frac{\alpha}{4}\left\|\theta_{\xi}\right\|_{L^{2}(0, T ; V)}^{2}+C_{1}\|f\|_{L^{\infty}\left(0, T ; V^{\prime}\right)}^{2}\|f\|_{L^{2}\left(0, T ; L^{q}(\Omega)\right)}^{2}, \\
\leq & \int_{0}^{t}\|f\|_{V^{\prime}} \cdot\left\|u_{\xi}\right\|_{L^{\infty}(\Omega)} \cdot\left\|\theta_{\xi}\right\|_{V} d t \\
\leq & \frac{\alpha}{4}\left\|\theta_{\xi}\right\|_{L^{2}(0, T ; V)}^{2}+C_{2}\|f\|_{L^{\infty}\left(0, T ; V^{\prime}\right)}^{2}\|f\|_{L^{2}\left(0, T ; L^{q}(\Omega)\right)}^{2},
\end{aligned}
$$

Hence, (25) follows

$$
\begin{aligned}
& \left\|\theta_{\xi}\right\|_{L^{2}(\Omega)}^{2}+\int_{0}^{t}\left\|\nabla \theta_{\xi}\right\|_{L^{2}(\Omega)}^{2} d t \\
\leq & C_{3}\left[\min \left(\frac{1}{2}, \frac{\alpha}{2}\right)\right]^{-1}\left\{\|f\|_{L^{\infty}\left(0, T ; V^{\prime}\right)}^{2}\|f\|_{L^{2}\left(0, T ; L^{q}(\Omega)\right)}^{2}+\left\|\theta_{0}\right\|_{L^{2}(\Omega)}^{2}\right\} .
\end{aligned}
$$

And again, choosing $v \in L^{2}(0, T ; V),\|v\|_{L^{2}(0, T ; V)}=1$ in equation (23), one easily deduce

$$
\left\|\theta_{\xi_{t}}\right\|_{L^{2}\left(0, T ; V^{\prime}\right)} \leq C\left\{\|f\|_{L^{\infty}\left(0, T ; V^{\prime}\right)}\|f\|_{L^{2}\left(0, T ; L^{q}(\Omega)\right)}+\left\|\theta_{0}\right\|_{L^{2}(\Omega)}\right\} .
$$


Therefore, provided we take $R$ large enough, $\xi \rightarrow \theta_{\xi}$ maps the ball $B_{R}$ of center 0 and radius $R$ in $L^{2}\left(0, T ; L^{2}(\Omega)\right)$ into itself. Moreover, since

$$
W=\left\{\theta \in L^{2}(0, T ; V) \mid \theta_{t} \in L^{2}\left(0, T ; V^{\prime}\right)\right\}
$$

is compactly imbedded in $L^{2}\left(0, T ; L^{2}(\Omega)\right)$, and this mapping will be carried into a relatively compact set by (28), (29). We are then going to show that this map is continuous, it will be done by the Schauder fixed point theorem. We consider a sequence $\xi_{n} \in L^{2}\left(0, T ; L^{2}(\Omega)\right)$ such that

$$
\xi_{n} \rightarrow \xi \quad \text { in } B_{R}
$$

defines $u_{\xi_{n}}$ as in (22) and $\theta_{\xi_{n}}=J\left(\xi_{n}\right)$. We will show that

$$
\theta_{\xi_{n}}=J\left(\xi_{n}\right) \rightarrow J(\xi)=\theta_{\xi} \quad \text { in } B_{R} .
$$

For that, subtracting the equation satisfied by $\theta_{\xi}$ from the one satisfied by $\theta_{\xi_{n}}$ with $\eta=\theta_{\xi_{n}}-\theta_{\xi}$, we get

$$
\begin{aligned}
\left(\frac{d}{d t}\left(\theta_{\xi_{n}}-\theta_{\xi}\right), \theta_{\xi_{n}}-\theta_{\xi}\right)+\left(k\left(\xi_{n}\right) \nabla \theta_{\xi_{n}}-k(\xi) \nabla \theta_{\xi}, \nabla\left(\theta_{\xi_{n}}-\theta_{\xi}\right)\right) \\
\quad+\left(\boldsymbol{b} \cdot \nabla\left(\theta_{\xi_{n}}-\theta_{\xi}\right), \theta_{\xi_{n}}-\theta_{\xi}\right)+\left(c\left(\theta_{\xi_{n}}-\theta_{\xi}\right), \theta_{\xi_{n}}-\theta_{\xi}\right) \\
=\left(\sigma\left(\xi_{n}\right)\left|\nabla u_{\xi_{n}}\right|^{2}-\sigma(\xi)\left|\nabla u_{\xi}\right|^{2}, \theta_{\xi_{n}}-\theta_{\xi}\right) .
\end{aligned}
$$

By (6), (14), (15) and (17), if integrating in $t$, we have,

$$
\begin{aligned}
& \quad \frac{1}{2}\left\|\theta_{\xi_{n}}-\theta_{\xi}\right\|_{L^{2}(\Omega)}^{2}+\alpha \int_{0}^{t}\left\|\nabla\left(\theta_{\xi_{n}}-\theta_{\xi}\right)\right\|_{L^{2}(\Omega)}^{2} d t \\
& \leq \frac{1}{2}\left\|\theta_{\xi_{n}}-\theta_{\xi}\right\|_{L^{2}(\Omega)}^{2}+\int_{0}^{t}\left(k\left(\xi_{n}\right) \nabla\left(\theta_{\xi_{n}}-\theta_{\xi}\right), \nabla\left(\theta_{\xi_{n}}-\theta_{\xi}\right)\right) d t \\
& \quad-\frac{1}{2} \int_{0}^{t}\left(\nabla \cdot \boldsymbol{b}\left(\theta_{\xi_{n}}-\theta_{\xi}\right), \theta_{\xi_{n}}-\theta_{\xi}\right) d t \\
& \quad+\int_{0}^{t}\left(c\left(\theta_{\xi_{n}}-\theta_{\xi}\right), \theta_{\xi_{n}}-\theta_{\xi}\right) d t \\
& =\int_{0}^{t}\left(\left(k(\xi)-k\left(\xi_{n}\right)\right) \nabla \theta_{\xi}, \nabla\left(\theta_{\xi_{n}}-\theta_{\xi}\right)\right) d t \\
& \quad+\int_{0}^{t}\left(\left(\sigma\left(\xi_{n}\right) u_{\xi_{n}} \nabla u_{\xi_{n}}-\sigma(\xi) u_{\xi} \nabla u_{\xi}\right), \nabla\left(\theta_{\xi_{n}}-\theta_{\xi}\right)\right) d t \\
& \quad+\int_{0}^{t}\left(f u_{\xi_{n}}-f u_{\xi}, \theta_{\xi_{n}}-\theta_{\xi}\right) d t \\
& =I_{1}+I_{2}+I_{3} .
\end{aligned}
$$


Set

$$
I_{4}=\frac{\alpha}{6} \int_{0}^{t}\left\|\nabla\left(\theta_{\xi_{n}}-\theta_{\xi}\right)\right\|_{L^{2}(\Omega)}^{2} d t,
$$

then by Hölder inequality and Poincaré inequality, we get

$$
\begin{aligned}
\left|I_{1}\right| & =\left|\int_{0}^{t}\left(\left(k(\xi)-k\left(\xi_{n}\right)\right) \nabla \theta_{\xi}, \nabla\left(\theta_{\xi_{n}}-\theta_{\xi}\right)\right) d t\right| \\
& \leq I_{4}+\frac{1}{\alpha} \int_{0}^{t}\left\|\left(k\left(\xi_{n}\right)-k(\xi)\right) \nabla \theta_{\xi}\right\|_{L^{2}(\Omega)}^{2} d t, \\
\left|I_{2}\right| & =\left|\int_{0}^{t}\left(\left(\sigma\left(\xi_{n}\right) u_{\xi_{n}} \nabla u_{\xi_{n}}-\sigma(\xi) u_{\xi} \nabla u_{\xi}\right), \nabla\left(\theta_{\xi_{n}}-\theta_{\xi}\right)\right) d t\right| \\
& \leq I_{4}+\frac{1}{\alpha} \int_{0}^{t}\left\|\sigma\left(\xi_{n}\right) u_{\xi_{n}} \nabla u_{\xi_{n}}-\sigma(\xi) u_{\xi} \nabla u_{\xi}\right\|_{L^{2}(\Omega)}^{2} d t, \\
\left|I_{3}\right| & =\left|\int_{0}^{t}\left(f u_{\xi_{n}}-f u_{\xi}, \theta_{\xi_{n}}-\theta_{\xi}\right) d t\right| \\
& \leq \int_{0}^{t}\|f\|_{V^{\prime}} \cdot\left\|u_{\xi_{n}}-u_{\xi}\right\|_{L^{\infty}(\Omega)} \cdot\left\|\theta_{\xi_{n}}-\theta_{\xi}\right\|_{V} d t \\
& \leq I_{4}+\frac{\|f\|_{L^{\infty}\left(0, T ; V^{\prime}\right)}^{2}}{\alpha} \int_{0}^{t}\left\|u_{\xi_{n}}-u_{\xi}\right\|_{L^{\infty}(\Omega)}^{2} d t .
\end{aligned}
$$

Thus, taking into account the Definition of $I_{4}$, we have

$$
\begin{aligned}
& \left\|\left(\theta_{\xi_{n}}-\theta_{\xi}\right)(t)\right\|_{L^{2}(\Omega)}^{2}+\int_{0}^{t}\left\|\nabla\left(\theta_{\xi_{n}}-\theta_{\xi}\right)\right\|_{L^{2}(\Omega)}^{2} d t \\
\leq & \frac{1}{\alpha}\left[\min \left(\frac{1}{2}, \frac{\alpha}{2}\right)\right]^{-1}\left\{\int_{0}^{T}\left\|\left(k\left(\xi_{n}\right)-k(\xi)\right) \nabla \theta_{\xi}\right\|_{L^{2}(\Omega)}^{2} d t\right. \\
& \left.+\int_{0}^{T}\left\|\sigma\left(\xi_{n}\right) u_{\xi_{n}} \nabla u_{\xi_{n}}-\sigma(\xi) u_{\xi} \nabla u_{\xi}\right\|_{L^{2}(\Omega)}^{2} d t\right\} \\
& +\frac{\|f\|_{L^{\infty}\left(0, T ; V^{\prime}\right)}^{2}}{\alpha}\left[\min \left(\frac{1}{2}, \frac{\alpha}{2}\right)\right]^{-1}\left\{\int_{0}^{T}\left\|u_{\xi_{n}}-u_{\xi}\right\|_{L^{\infty}(\Omega)}^{2} d t\right\} .
\end{aligned}
$$

Since $\theta_{\xi_{n}}$ is in a relatively compact set of $B_{R}$, it is enough to show that $\theta_{\xi}$ is the only limit point for $\theta_{\xi_{n}}$. Let $\theta_{\xi}^{\prime}$ be such limit point, i.e.

$$
\theta_{\xi}^{\prime}=\lim _{n_{m} \rightarrow \infty} \theta_{\xi} \quad \text { in } B_{R}
$$

provided that we have extracted another sequence of $n_{m}$ that still denoted by $n_{m}$ we can assume

$$
\xi_{n m} \rightarrow \xi \quad \text { a.e. in } \Omega \times(0, T) .
$$


Then, since $\left|\nabla \theta_{\xi}\right|^{2} \in L^{1}\left(Q_{T}\right)$, and by (6), we know almost everywhere by the Lebesgue theorem,

$\int_{0}^{T}\left\|\left(k\left(\xi_{n_{m}}\right)-k(\xi)\right) \nabla \theta_{\xi}\right\|_{L^{2}(\Omega)}^{2} d t=\int_{0}^{T} \int_{\Omega}\left|k\left(\xi_{n m}\right)-k(\xi)\right|^{2}\left|\nabla \theta_{\xi}\right|^{2} d x d t \rightarrow 0$.

Next, for $n=n_{m}$ the second integral in the right-hand side of (35) reads

$$
\begin{aligned}
& \int_{0}^{T}\left\|\sigma\left(\xi_{n}\right) u_{\xi_{n}} \nabla u_{\xi_{n}}-\sigma(\xi) u_{\xi} \nabla u_{\xi}\right\|_{L^{2}(\Omega)}^{2} d t \\
\leq & \int_{0}^{T}\left\|\sigma\left(\xi_{n}\right) u_{\xi_{n}} \nabla u_{\xi_{n}}-\sigma\left(\xi_{n}\right) u_{\xi_{n}} \nabla u_{\xi}\right\|_{L^{2}(\Omega)}^{2} d t \\
& +\int_{0}^{T}\left\|\sigma\left(\xi_{n}\right) u_{\xi_{n}} \nabla u_{\xi}-\sigma\left(\xi_{n}\right) u_{\xi} \nabla u_{\xi}\right\|_{L^{2}(\Omega)}^{2} d t \\
& +\int_{0}^{T}\left\|\sigma\left(\xi_{n}\right) u_{\xi} \nabla u_{\xi}-\sigma(\xi) u_{\xi} \nabla u_{\xi}\right\|_{L^{2}(\Omega)}^{2} d t \\
=I & +I I+I I I .
\end{aligned}
$$

It is clear that

$$
\begin{aligned}
I & \leq C \int_{0}^{T}\|f\|_{L^{q}(\Omega)}^{2}\left\|\nabla\left(u_{\xi_{n}}-u_{\xi}\right)\right\|_{L^{2}(\Omega)}^{2} d t, \\
I I & \leq C \int_{0}^{T}\left\|\left(u_{\xi_{n}}-u_{\xi}\right) \nabla u_{\xi}\right\|_{L^{2}(\Omega)}^{2} d t, \\
I I I & \leq C \int_{0}^{T}\|f\|_{L^{q}(\Omega)}^{2}\left\|\left(\sigma\left(\xi_{n}\right)-\sigma(\xi)\right) \nabla u_{\xi}\right\|_{L^{2}(\Omega)}^{2} d t .
\end{aligned}
$$

By (36), (6) and Lemma 1, together with the Lebesgue theorem we can obtain $I I I \rightarrow 0$. Next, $u_{\xi_{n}}$ satisfies

$$
-\nabla \cdot\left(\sigma\left(\xi_{n}\right) \nabla u_{\xi_{n}}\right)=f ; \quad u_{\xi_{n}}=0 \quad \text { on } \Gamma,
$$

hence

$$
\left(\sigma\left(\xi_{n}\right) \nabla u_{\xi_{n}}, \nabla\left(u_{\xi_{n}}-u_{\xi}\right)\right)=\left(\sigma(\xi) \nabla u_{\xi}, \nabla\left(u_{\xi_{n}}-u_{\xi}\right)\right)
$$

or

$$
\left(\sigma\left(\xi_{n}\right) \nabla\left(u_{\xi_{n}}-u_{\xi}\right), \nabla\left(u_{\xi_{n}}-u_{\xi}\right)\right)=\left(\left(\sigma(\xi)-\sigma\left(\xi_{n}\right)\right) \nabla u_{\xi}, \nabla\left(u_{\xi_{n}}-u_{\xi}\right)\right),
$$

which implies, for every $t$,

$$
\left\|\nabla\left(u_{\xi_{n}}-u_{\xi}\right)\right\|_{L^{2}(\Omega)}^{2} \leq C\left\|\left(\sigma(\xi)-\sigma\left(\xi_{n}\right)\right) \nabla u_{\xi}\right\|_{L^{2}(\Omega)}^{2} .
$$


Thus,

$$
I \leq C \int_{0}^{T}\|f\|_{L^{q}(\Omega)}^{2}\left\|\left(\sigma\left(\xi_{n}\right)-\sigma(\xi)\right) \nabla u_{\xi}\right\|_{L^{2}(\Omega)}^{2} d t \rightarrow 0
$$

as above for $I I I$. By the Poincaré inequality, this implies

$$
\int_{0}^{T}\left\|u_{\xi_{n}}-u_{\xi}\right\|_{L^{2}(\Omega)}^{2} d t \rightarrow 0,
$$

and up to an extracted subsequence we can assume

$$
u_{\xi_{n}}-u_{\xi} \rightarrow 0 \quad \text { a.e. on } \Omega \times(0, T) ;
$$

then the Lebesgue convergence theorem gives $I I \rightarrow 0$, which also implies the third integral in the right-hand side of (35) approaches to zero almost everywhere in $\Omega \times(0, T)$. Hence $\theta_{\xi} \rightarrow \theta_{\xi}=\theta_{\xi}^{\prime}$ in $L^{2}\left(0, T ; L^{2}(\Omega)\right)$.

\section{Uniqueness of the solution}

Definition 1. We denote by $\mathcal{R}_{s}$ for $1<s<\infty$ the class of regular subsets $G$ in $\mathbb{R}^{d}$ for which the Laplacian operator maps $W_{0}^{1, s}(G)$ onto $W^{-1, s}(G)$.

Remark 1. A bounded $C^{1}$ domain, for example, is of class $\mathcal{R}_{s}$ for every $s \in$ $(2, \infty)$, see Theorem 4.6 in [34].

From now on, we assume that $\Omega$ is of class $\mathcal{R}_{r^{*}}$ for some $r^{*}>2$. For $1 \leq s \leq r^{*}$, we define $M_{s} \geq 1$ by

$$
\inf _{u \in W_{0}^{1, s} \backslash\{0\}} \sup _{v \in W_{0}^{1, s^{\prime}} \backslash\{0\}} \frac{(\nabla u, \nabla v)}{\|\nabla u\|_{L^{s}(\Omega)}\|\nabla v\|_{L^{s^{\prime}}(\Omega)}}=\frac{1}{M_{S}} .
$$

It is easy to see that $M_{2}=1$ and $M_{s^{\prime}}=M_{s}$.

Lemma 2. ([32]) If $r \in\left(2, r^{*}\right]$ is such that

$$
M_{r} \frac{\sigma_{2}-\sigma_{1}}{\sigma_{1}+\sigma_{2}}<1,
$$

then, for any $\theta$, we have

$$
\inf _{u \in W_{0}^{1, r} \backslash\{0\}} \sup _{v \in W_{0}^{1, r^{\prime}} \backslash\{0\}} \frac{a(\theta ; u, v)}{\|\nabla u\|_{L^{r}(\Omega)}\|\nabla v\|_{L^{r^{\prime}(\Omega)}}} \geq \gamma
$$


where

$$
\gamma=\frac{\sigma_{1}+\sigma_{2}}{2 M_{r}}\left(1-M_{r} \frac{\sigma_{2}-\sigma_{1}}{\sigma_{1}+\sigma_{2}}\right)>0 .
$$

Similarly to $[24,32]$, we have

Lemma 3. Let $f \in L^{\infty}\left(0, T ; W^{-1, r}(\Omega)\right)$, where $r$ is defined in Lemma 2. Then, for any given $\theta$, the solution to (13.i) $u \in L^{\infty}\left(0, T ; W_{0}^{1, r}(\Omega)\right)$ and satisfies that

$$
\|\nabla u\|_{L^{\infty}\left(0, T ; L^{r}(\Omega)\right)} \leq \frac{1}{\gamma}\|f\|_{L^{\infty}\left(0, T ; W^{-1, r}(\Omega)\right)}
$$

where $\gamma$ is defined by (43).

Lemma 4. Under the assumptions of Lemma 3 , if $\boldsymbol{b}, c$ are bounded continuous functions, $\sigma, k \in L^{\infty}(\mathbb{R})$, then solution to (13.ii) $\theta \in W^{r}$ with $\theta(0)=\theta_{0}$, where

$$
W^{r}=\left\{\theta \in L^{r}\left(0, T ; W^{1, r}(\Omega)\right): \theta_{t} \in L^{r}\left(0, T ; W^{-1, r}(\Omega)\right)\right\} .
$$

Proof. Since $u \in L^{\infty}\left(0, T ; W^{1, r}(\Omega)\right)$, it follows that $\sigma(\theta)|\nabla u|^{2} \in L^{\infty}(0, T$; $\left.L^{r / 2}(\Omega)\right) \hookrightarrow L^{\infty}\left(0, T ; W^{-1, r}(\Omega)\right) \hookrightarrow L^{r}\left(0, T ; W^{-1, r}(\Omega)\right)$. Following the idea of Theorem 1 and Remark 5 in [21], and the similar proof in Chapter 4 of [18] we can complete the proof.

To study the uniqueness of problem (13), we need to assume that: $\sigma, k$ are Lipschitz continuous, i.e. there is a Lipschitz constant $L$, for any $\xi_{1}, \xi_{2} \in \mathbb{R}$ such that,

$$
\left|k\left(\xi_{1}\right)-k\left(\xi_{2}\right)\right|, \quad\left|\sigma\left(\xi_{1}\right)-\sigma\left(\xi_{2}\right)\right| \leq L\left|\xi_{1}-\xi_{2}\right| .
$$

Let $\left(u_{i}, \theta_{i}\right), i=1,2$, two weak solutions to problem (13), and set $\bar{\theta}=\theta_{1}-$ $\theta_{2}, \bar{u}=u_{1}-u_{2}$, noticing (4), we have $\forall v \in V$,

$$
a\left(\theta_{1} ; \bar{u}, v\right)=a\left(\theta_{1} ; u_{1}, v\right)-a\left(\theta_{1} ; u_{2}, v\right)=a\left(\theta_{2} ; u_{2}, v\right)-a\left(\theta_{1} ; u_{2}, v\right) .
$$

Therefore, letting $v=\bar{u}$, by (7) and (45), we have

$$
\sigma_{1}\|\nabla \bar{u}\|_{L^{2}(\Omega)}^{2} \leq L\|\nabla \bar{u}\|_{L^{2}(\Omega)}\left\|\nabla u_{2}\right\|_{L^{r}(\Omega)}\|\bar{\theta}\|_{L^{2 r /(r-2)}(\Omega)}
$$

or

$$
\|\nabla \bar{u}\|_{L^{2}(\Omega)} \leq C\left\|\nabla u_{2}\right\|_{L^{r}(\Omega)}\|\bar{\theta}\|_{L^{2 r /(r-2)}(\Omega)} .
$$


On the other hand, subtracting the equation satisfied by $\theta_{1}$ from the one satisfied by $\theta_{2}$, we get, for any $\eta \in V$,

$$
\begin{aligned}
& \left(\bar{\theta}_{t}, \eta\right)+b\left(\theta_{1} ; \bar{\theta}, \eta\right) \\
= & {\left[b\left(\theta_{2} ; \theta_{2}, \eta\right)-b\left(\theta_{1} ; \theta_{2}, \eta\right)\right]+\left(\sigma\left(\theta_{1}\right)\left|\nabla u_{1}\right|^{2}-\sigma\left(\theta_{2}\right)\left|\nabla u_{2}\right|^{2}, \eta\right) } \\
= & \left(\left[k\left(\theta_{2}\right)-k\left(\theta_{1}\right)\right] \nabla \theta_{2}, \nabla \eta\right)+\left(\left[\sigma\left(\theta_{1}\right)-\sigma\left(\theta_{2}\right)\right]\left|\nabla u_{1}\right|^{2}\right. \\
& \left.+\sigma\left(\theta_{2}\right) \nabla \bar{u} \cdot \nabla\left(u_{1}+u_{2}\right), \eta\right) .
\end{aligned}
$$

Let $\eta=\bar{\theta}$, and noticing (17), we have

$$
\begin{aligned}
& \frac{1}{2} \frac{d}{d t}\|\bar{\theta}(t)\|_{L^{2}(\Omega)}^{2}+\alpha\|\nabla \bar{\theta}\|_{L^{2}(\Omega)}^{2} \\
\leq & \left(\left(k\left(\theta_{1}\right)-k\left(\theta_{2}\right)\right) \nabla \theta_{2}, \nabla \bar{\theta}\right)+\left(\left(\sigma\left(\theta_{1}\right)-\sigma\left(\theta_{2}\right)\right)\left|\nabla u_{1}\right|^{2}, \bar{\theta}\right) \\
& +\left(\sigma\left(\theta_{2}\right) \nabla \bar{u} \cdot \nabla\left(u_{1}+u_{2}\right), \bar{\theta}\right) .
\end{aligned}
$$

By Hölder and Young inequalities, we easily can deduce that

$$
\begin{aligned}
\left.\mid\left(k\left(\theta_{1}\right)-k\left(\theta_{2}\right)\right) \nabla \theta_{2}, \nabla \bar{\theta}\right) \mid & \leq L\left\|\nabla \theta_{2}\right\|_{L^{r}(\Omega)}\|\nabla \bar{\theta}\|_{L^{2}(\Omega)}\|\bar{\theta}\|_{L^{2 r /(r-2)}(\Omega)} \\
& \leq \varepsilon\|\nabla \bar{\theta}\|_{L^{2}(\Omega)}^{2}+C_{\varepsilon}\left\|\nabla \theta_{2}\right\|_{L^{r}(\Omega)}^{2}\|\bar{\theta}\|_{L^{2 r /(r-2)}(\Omega)}^{2}, \\
\left|\left(\left(\sigma\left(\theta_{1}\right)-\sigma\left(\theta_{2}\right)\right)\left|\nabla u_{1}\right|^{2}, \bar{\theta}\right)\right| & \leq L\left\|\nabla u_{1}\right\|_{L^{r}(\Omega)}^{2}\|\bar{\theta}\|_{L^{2 r /(r-2)}(\Omega)}^{2}, \\
\left|\left(\sigma\left(\theta_{2}\right) \nabla \bar{u} \cdot \nabla\left(u_{1}+u_{2}\right), \bar{\theta}\right)\right| & \leq \sigma_{2}\|\nabla \bar{u}\|_{L^{2}(\Omega)}\left\|\nabla\left(u_{1}+u_{2}\right)\right\|_{L^{r}(\Omega)}\|\bar{\theta}\|_{L^{2 r /(r-2)}(\Omega)} .
\end{aligned}
$$

Combining the above estimates and (46), choosing $\varepsilon=\frac{\alpha}{4}$, (47) follows

$$
\begin{aligned}
& \frac{1}{2} \frac{d}{d t}\|\bar{\theta}\|_{L^{2}(\Omega)}^{2}+\frac{3 \alpha}{4}\|\nabla \bar{\theta}\|_{L^{2}(\Omega)}^{2} \\
\leq & C\left(\left\|\nabla \theta_{2}\right\|_{L^{r}(\Omega)}^{2}+\left\|\nabla u_{1}\right\|_{L^{r}(\Omega)}^{2}+\left\|\nabla u_{2}\right\|_{L^{r}(\Omega)}^{2}\right)\|\bar{\theta}\|_{L^{2 r(r-2)}(\Omega)}^{2} .
\end{aligned}
$$

By the Galiardo-Nirenberg interpolation inequality, (48) becomes

$$
\begin{aligned}
& \frac{1}{2} \frac{d}{d t}\|\bar{\theta}\|_{L^{2}(\Omega)}^{2}+\frac{3 \alpha}{4}\|\nabla \bar{\theta}\|_{L^{2}(\Omega)}^{2} \\
\leq & C\left(\left\|\nabla \theta_{2}\right\|_{L^{r}(\Omega)}^{2}+\left\|\nabla u_{1}\right\|_{L^{r}(\Omega)}^{2}+\left\|\nabla u_{2}\right\|_{L^{r}(\Omega)}^{2}\right)\|\bar{\theta}\|_{L^{2}(\Omega)}^{2\left(1-\frac{d}{r}\right)}\|\nabla \bar{\theta}\|_{L^{2}(\Omega)}^{\frac{2 d}{r}} \\
\leq & \varepsilon\|\nabla \bar{\theta}\|_{L^{2}(\Omega)}^{2}+C_{\varepsilon}\left(\left\|\nabla \theta_{2}\right\|_{L^{r}(\Omega)}^{2 r(r-d)}+\left\|\nabla u_{1}\right\|_{L^{r}(\Omega)}^{2 r /(r-d)}+\left\|\nabla u_{2}\right\|_{L^{r}(\Omega)}^{2 r /(r-d)}\right)\|\bar{\theta}\|_{L^{2}(\Omega)}^{2}
\end{aligned}
$$


where we apply Young's inequality $a b \leq \varepsilon a^{r / d}+C_{\varepsilon} b^{r /(r-d)}$. Again choosing $\varepsilon=\frac{\alpha}{4}$, and by the estimates in Lemma 3, 4, and by Sobolev inequality that $L^{r}(0, T) \subset L^{2 r /(r-d)}(0, T)$, Gronwall lemma implies that

$$
\|\bar{\theta}(t)\|_{L^{2}(\Omega)}^{2}+\int_{0}^{t}\|\nabla \bar{\theta}(\tau)\|_{L^{2}(\Omega)}^{2} d \tau \leq C\|\bar{\theta}(0)\|_{L^{2}(\Omega)}^{2} .
$$

Thus, by (46), uniqueness of the solution follows. Therefore, with the above result, we can state:

Theorem 2. (Uniqueness) Under the conditions of Lemmas 3 and 4 with Lipschitz assumption on $k, \sigma$ as (45), there exists at most one weak solution to problem (2).

Remark 2. Here the uniqueness result is obtained without additional regularity assumptions on the solution as those required in [10].

\section{Regularity of the solution}

In this section we study the regularity of the solution to the problem (2) only on the dimension of space $d \leq 2$ ( in [33] we considered a simplified case of $k(\theta) \equiv 1)$. For our regularity estimates, we need to assume that

$$
\sigma(s), k(s) \in C^{2}(\mathbb{R}), \quad\left|\sigma^{\prime}(s)\right|+\left|\sigma^{\prime \prime}(s)\right|+\left|k^{\prime}(s)\right|+\left|k^{\prime \prime}(s)\right| \leq L^{\prime}
$$

for all $s \in \mathbb{R}$, where $L^{\prime}$ is some positive constant.

Then we have:

Theorem 3. (Regularity) Let $T>0$ and assume that $\theta_{0} \in H^{2}(\Omega) \cap H_{0}^{1}(\Omega)$, $\boldsymbol{b}, c$ are bounded continuous functions. $f \in C^{2}\left(0, T ; L^{r}(\Omega)\right)$ where $r$ is defined in Lemma 2. Then problem (2) has a unique solution $u \in L^{\infty}\left(0, T ; H^{2}(\Omega)\right)$, $\theta \in C^{1}\left(0, T ; L^{2}(\Omega)\right) \cap C\left(0, T ; H^{2}(\Omega)\right)$. Moreover, there is a constant $C$, depending on $T, \theta_{0}, f, \Omega$ and on $\sigma, k$ through the constants $\sigma_{i}, k_{i}$ in (6), such that for every $t \in[0, T]$, we have

$$
\begin{aligned}
\|u(t)\|_{H^{2}(\Omega)} & +\|u(t)\|_{W^{1, \infty}(\Omega)}+\left\|u_{t}(t)\right\|_{H^{1}(\Omega)}+\|\theta(t)\|_{H^{2}(\Omega)} \\
& +\left\|\theta_{t}(t)\right\|_{L^{2}(\Omega)}+t\left\|\theta_{t}(t)\right\|_{H^{1}(\Omega)}+t\left\|\theta_{t t}(t)\right\|_{L^{2}(\Omega)} \leq C
\end{aligned}
$$


Proof. We consider the initial value problem ( here $V=H^{2}(\Omega) \cap H_{0}^{1}(\Omega)$ )

$$
\begin{cases}\theta(t) \in V, & \\ \left(\theta_{t}, \eta\right)+(k(\theta) \nabla \theta, \nabla \eta)+(\boldsymbol{b} \cdot \nabla \theta, \eta)+(c \theta, \eta) & \\ =\left(\sigma(\theta)|\nabla u|^{2}, \eta\right), & \forall \eta \in V, t>0 \\ (\theta(0), \eta)=\left(\theta_{0}, \eta\right), & \forall \eta \in V\end{cases}
$$

where $u(t)$ is determined by the linear elliptic problem

$$
u(t) \in H_{0}^{1}, \quad(\sigma(\theta) \nabla u, \nabla v)=(f, v) \quad \forall v \in H_{0}^{1}, t>0
$$

we are now to analyze the regularity of the solution.

Step 1. We first show some estimates of $u$. By (10), we know

$$
\|u\|_{L^{\infty}(\Omega)} \leq C\|f\|_{L^{q}(\Omega)}
$$

for some $q>d / 2$. Next, by Lemma 3, there existes a $2<r<r^{*}$ such that $\|\nabla u(t)\|_{L^{r}(\Omega)} \leq C\|f\|_{W^{-1, r}(\Omega)}$. This implies that for every $t$,

$$
\|u(t)\|_{W^{1, r}(\Omega)} \leq C\|f\|_{L^{r}(\Omega)} \leq C
$$

We note that we should consider mostly the derivatives of $\sigma(\theta)$ to obtain further estimates of $u$. First, equation (51) implies that $-\sigma(\theta) \Delta u-\nabla \sigma(\theta) \cdot \nabla u=f$, so that by Hölder inequality,

$$
\|\Delta u\|_{L^{r}(\Omega)}=\left\|\frac{f+\nabla \sigma(\theta) \cdot \nabla u}{\sigma(\theta)}\right\|_{L^{r}(\Omega)} \leq C\|f\|_{L^{r}(\Omega)}+C\|\nabla \theta\|_{L^{r_{1}(\Omega)}}\|\nabla u\|_{L^{r_{2}(\Omega)}}
$$

for any $r_{1}, r_{2}$ satisfying $\frac{1}{r_{1}}+\frac{1}{r_{2}}=\frac{1}{r}$. We thus obtain

$$
\|u\|_{W^{2, r}(\Omega)} \leq C\|\Delta u\|_{L^{r}(\Omega)} \leq C\left(\|f\|_{L^{r}(\Omega)}+\|\nabla \theta\|_{L^{r_{1}(\Omega)}}\|\nabla u\|_{L^{r_{2}(\Omega)}}\right) .
$$

Hence, by Gagliardo-Nirenberg interpolation inequality and estimate (53), we will get

$$
\begin{aligned}
\|u\|_{W^{2, r}(\Omega)} & \leq C\left(\|f\|_{L^{r}(\Omega)}+\|\theta\|_{H^{2}(\Omega)}\|u\|_{W^{1, r}(\Omega)}^{\delta}\|u\|_{W^{2, r}(\Omega)}^{1-\delta}\right) \\
& \leq C\left(\|f\|_{L^{r}(\Omega)}+\|\theta\|_{H^{2}(\Omega)}\|u\|_{W^{2, r}(\Omega)}^{1-\delta}\right) \\
& \leq\left(\|f\|_{L^{r}(\Omega)}+\|\theta\|_{H^{2}(\Omega)}^{1 / \delta}\right)+\frac{1}{2}\|u\|_{W^{2, r}(\Omega)}
\end{aligned}
$$


where $\delta=1-d / r+d / r_{2}=1-d / r_{1}$. In the last step we also used Young's inequality

$$
a b \leq \varepsilon^{1-1 / \delta} a^{1 / \delta}+\varepsilon b^{1 /(1-\delta)}, \quad \varepsilon>0,0<\delta<1, a, b>0
$$

For the above estimates of $\|\nabla \theta\|_{L^{r_{1}(\Omega)}}$ to hold, it is required that $r_{1}<\infty$, which in its turn is equivalent to $\delta<1$. Thus, we have proved the preliminary estimate

$$
\|u(t)\|_{H^{r}(\Omega)} \leq C\left(1+\|\theta(t)\|_{H^{2}(\Omega)}^{1 / \delta}\right), \quad \forall \delta \in[1-d / p, 1)
$$

where $C$ is independent of $\delta$. Next, arguing as [16] treats, we get

$$
\|u(t)\|_{W^{1, \infty}(\Omega)}+\|u(t)\|_{W^{1,4}(\Omega)}^{2} \leq C\left(1+\|\theta(t)\|_{H^{2}(\Omega)}^{\rho}\right)
$$

where $\rho<1$ if $d \leq 2$.

Step 2. We now estimates $\|\theta\|_{H^{2}(\Omega)}$ and $\left\|\theta_{t}\right\|_{L^{2}(\Omega)}$. First, without lost of generality, we assume that $\|\theta\|_{W^{1,4}(\Omega)} \leq C$ (see Lemma 4). We note that it suffices to estimate $\left\|\theta_{t}\right\|_{L^{2}(\Omega)}$. In fact, equation (50) implies that $\theta_{t}-k(\theta) \Delta \theta+\boldsymbol{b} \cdot \nabla \theta+$ $c \theta=P\left(\sigma(\theta)|\nabla u|^{2}+k^{\prime}(\theta)|\nabla \theta|^{2}\right)$, where $P$ denotes the orthogonal projection onto $V$. Hence,

$$
\begin{aligned}
\|\theta\|_{H^{2}(\Omega)} \leq & C\|\Delta \theta\|_{L^{2}(\Omega)} \leq C\left(\left\|\theta_{t}\right\|_{L^{2}(\Omega)}+\|\boldsymbol{b} \cdot \nabla \theta\|_{L^{2}(\Omega)}+\|c \theta\|_{L^{2}(\Omega)}\right. \\
& \left.+\left\|\sigma(\theta)|\nabla u|^{2}\right\|_{L^{2}(\Omega)}+\left\|k^{\prime}(\theta)|\nabla \theta|^{2}\right\|_{L^{2}(\Omega)}\right) \\
\leq & C\left(\left\|\theta_{t}\right\|_{L^{2}(\Omega)}+\|u\|_{W^{1,4}(\Omega)}^{2}+\|\theta\|_{W^{1,4}(\Omega)}^{2}+\|\nabla \theta\|_{L^{2}(\Omega)}+\|\theta\|_{L^{2}(\Omega)}\right) .
\end{aligned}
$$

By the interpolation inequality, we know the two terms in the last inequality can be estimaed by $\|\theta\|_{H^{2}(\Omega)}$, and in view of the estimate (55), so we can get

$$
\|\theta(t)\|_{H^{2}(\Omega)} \leq C\left(1+\left\|\theta_{t}(t)\right\|_{L^{2}(\Omega)}\right) .
$$

For the further estimates of $\theta_{t}$, we differentiate equations (50) and (51) with respect to $t$. Beginning with (51), we have

$$
\left(\sigma(\theta) \nabla u_{t}, \nabla v\right)=-\left(\sigma(\theta)_{t} \nabla u, \nabla v\right)+\left(f_{t}, v\right), \quad \forall v \in H_{0}^{1}
$$

Because $\sigma(\theta)$ is continuous in $C(\mathbb{R})$, and in view of (55), we have

$$
\begin{aligned}
\left\|u_{t}\right\|_{H^{1}(\Omega)} & \leq C\left(\left\|\sigma^{\prime}(\theta) \theta_{t} \nabla u\right\|_{L^{2}(\Omega)}+\left\|f_{t}\right\|_{L^{2}(\Omega)}\right) \\
& \leq C\left(1+\left\|\theta_{t}\right\|_{L^{2}(\Omega)}\|u\|_{W^{1, \infty}(\Omega)}\right) \\
& \leq C\left(1+\left\|\theta_{t}\right\|_{L^{2}(\Omega)}^{2}\right) .
\end{aligned}
$$


Next, by (12), if differentiating equation (50), we have

$$
\begin{aligned}
& \left(\theta_{t t}, \eta\right)+\left((k(\theta) \nabla \theta)_{t}, \nabla \eta\right)+\left(\boldsymbol{b} \cdot \nabla \theta_{t}, \eta\right)+\left(c \theta_{t}, \eta\right) \\
= & -\left((\sigma(\theta) u \nabla u)_{t}, \nabla \eta\right)+\left((f u)_{t}, \eta\right)
\end{aligned}
$$

for each $\eta \in V$, where $(k(\theta) \nabla \theta)_{t}=k^{\prime}(\theta) \theta_{t} \nabla \theta+k(\theta) \nabla \theta_{t}$. Similarly, if condition (15) is satisfied, with $\eta=\theta_{t}$ in above equation, we would get

$$
\begin{aligned}
& \frac{1}{2} \frac{d}{d t}\left\|\theta_{t}\right\|_{L^{2}(\Omega)}^{2}+\alpha\left\|\nabla \theta_{t}\right\|_{L^{2}(\Omega)}^{2} \\
\leq & \frac{1}{2} \frac{d}{d t}\left\|\theta_{t}\right\|_{L^{2}(\Omega)}^{2}+\left(k(\theta) \nabla \theta_{t}, \nabla \theta_{t}\right)-\frac{1}{2}\left(\nabla \cdot \boldsymbol{b} \theta_{t}, \theta_{t}\right)+\left(c \theta_{t}, \theta_{t}\right) \\
= & \left|\left((\sigma(\theta) u \nabla u)_{t}, \nabla \theta_{t}\right)\right|+\left|\left((f u)_{t}, \theta_{t}\right)\right|+\left|\left(k^{\prime}(\theta) \theta_{t} \nabla \theta, \nabla \theta_{t}\right)\right| \\
\leq & C\left(\left\|(\sigma(\theta) u \nabla u)_{t}\right\|_{L^{2}(\Omega)}^{2}+\left\|(f u)_{t}\right\|_{L^{2}(\Omega)}^{2}\right) \\
& +L^{\prime}\left\|\theta_{t}\right\|_{L^{4}(\Omega)}^{2}\|\nabla \theta\|_{L^{4}(\Omega)}^{2}+\varepsilon\left\|\nabla \theta_{t}\right\|_{L^{2}(\Omega)}^{2}
\end{aligned}
$$

where

$$
\begin{aligned}
& \left\|(\sigma(\theta) u \nabla u)_{t}\right\|_{L^{2}(\Omega)} \\
\leq & \left\|\sigma^{\prime}(\theta) \theta_{t} u \nabla u\right\|_{L^{2}(\Omega)}+\left\|\sigma(\theta) u_{t} \nabla u\right\|_{L^{2}(\Omega)}+\left\|\sigma(\theta) u \nabla u_{t}\right\|_{L^{2}(\Omega)} \\
\leq & C\left(\left\|\theta_{t}\right\|_{L^{2}(\Omega)}\|u\|_{L^{\infty}(\Omega)}\|u\|_{W^{1, \infty}(\Omega)}+\left\|u_{t}\right\|_{L^{\tilde{r}}(\Omega)}\|u\|_{W^{1, r}(\Omega)}\right. \\
& \left.+\|u\|_{L^{\infty}(\Omega)}\left\|u_{t}\right\|_{H^{1}(\Omega)}\right)
\end{aligned}
$$

$1 / r+1 / \tilde{r}=1 / 2$. By Sobolev's inequality and known bounds for $u$ and $u_{t}$ in (52), (53), (55), (58), we get $\left\|(\sigma(\theta) u \nabla u)_{t}\right\|_{L^{2}(\Omega)} \leq C\left(1+\left\|\theta_{t}\right\|_{L^{2}(\Omega)}^{2}\right)$, thus (60) follows

$$
\frac{d}{d t}\left\|\theta_{t}\right\|_{L^{2}(\Omega)}^{2}+\left\|\nabla \theta_{t}\right\|_{L^{2}(\Omega)}^{2} \leq C\left(1+\left\|\theta_{t}\right\|_{L^{2}(\Omega)}^{4}\right)
$$

Since $\theta(0)=P\left(\theta_{0}\right)$, thus

$$
\|\theta(0)\|_{H^{2}(\Omega)} \leq C\|\Delta \theta(0)\|_{L^{2}(\Omega)} \leq C\left\|\theta_{0}\right\|_{H^{2}(\Omega)} \leq C,
$$

then by (55),

$$
\begin{aligned}
\left\|\theta_{t}(0)\right\|_{L^{2}(\Omega)} \leq & \|k(\theta(0)) \Delta \theta(0)\|_{L^{2}(\Omega)}+\|\boldsymbol{b} \cdot \nabla \theta(0)\|_{L^{2}(\Omega)}+\|c \theta(0)\|_{L^{2}(\Omega)} \\
& +\left\|k^{\prime}(\theta(0))|\nabla \theta(0)|^{2}\right\|_{L^{2}(\Omega)}+\left\|P\left(\sigma(\theta(0))|\nabla u(0)|^{2}\right)\right\|_{L^{2}(\Omega)} \\
\leq & C\|\Delta \theta(0)\|_{L^{2}(\Omega)}+C\|u(0)\|_{W^{1,4}(\Omega)}^{2}+C\|\theta(0)\|_{W^{1,4}(\Omega)}^{2} \leq C
\end{aligned}
$$


Therefore, integrating (61) in $t$, we get

$$
\left\|\theta_{t}(t)\right\|_{L^{2}(\Omega)}^{2}+\int_{0}^{t}\left\|\nabla \theta_{t}(\tau)\right\|_{L^{2}(\Omega)}^{2} d \tau \leq C+C \int_{0}^{t}\left\|\theta_{t}\right\|_{L^{2}(\Omega)}^{4} d \tau
$$

By Gronwall's lemma, we obtain

$$
\left\|\theta_{t}(t)\right\|_{L^{2}(\Omega)}^{2}+\int_{0}^{t}\left\|\nabla \theta_{t}(\tau)\right\|_{L^{2}(\Omega)}^{2} d \tau \leq \exp \left(\int_{0}^{t}\left\|\theta_{t}(\tau)\right\|_{L^{2}(\Omega)}^{2} d \tau\right)
$$

We are now to establish the estimates of the right hand side of the above inequality. Taking $\eta=\theta_{t}$ in (50),

$$
\begin{aligned}
& \left\|\theta_{t}\right\|_{L^{2}(\Omega)}^{2}+\frac{1}{2} \frac{d}{d t}(k(\theta) \nabla \theta, \nabla \theta)+\left(\boldsymbol{b} \cdot \nabla \theta, \theta_{t}\right)+\left(c \theta, \theta_{t}\right) \\
= & \left(\sigma(\theta)|\nabla u|^{2}, \theta_{t}\right)+\frac{1}{2}\left(k^{\prime}(\theta)|\nabla \theta|^{2}, \theta_{t}\right) \\
\leq & C\|u\|_{W^{1,4}(\Omega)}^{2}\left\|\theta_{t}\right\|_{L^{2}(\Omega)}+\frac{L^{\prime}}{2}\|\nabla \theta\|_{L^{4}(\Omega)}^{2}\left\|\theta_{t}\right\|_{L^{2}(\Omega)}
\end{aligned}
$$

In view of (56) and Sobolev inbedding inequality, if integrating in $t$, we arrive at

$$
\int_{0}^{t}\left\|\theta_{t}\right\|_{L^{2}(\Omega)}^{2} d \tau+\|\nabla \theta\|_{L^{2}(\Omega)}^{2} \leq C\left\|\nabla P\left(\theta_{0}\right)\right\|_{L^{2}(\Omega)}^{2}+C t \leq C
$$

since $\theta(0)=P\left(\theta_{0}\right)$, where $P$ is bounded with respect to the norm $\|\nabla \cdot\|_{L^{2}(\Omega)}$, which implies (62) is bounded by $C$.

Substituting this result into (54),(55), (56), (58), we may conclude that

$$
\begin{aligned}
\|u(t)\|_{H^{r}(\Omega)} & +\|u(t)\|_{W^{1, \infty}(\Omega)}+\left\|u_{t}(t)\right\|_{H^{1}(\Omega)} \\
& +\|\theta(t)\|_{H^{2}(\Omega)}+\left\|\theta_{t}(t)\right\|_{L^{2}(\Omega)} \leq C
\end{aligned}
$$

Step 3. We next to estimate $t\left\|\theta_{t}(t)\right\|_{H^{2}(\Omega)}$ and $t\left\|\theta_{t t}(t)\right\|_{L^{2}(\Omega)}$. We note that $\theta_{t t}-\nabla \cdot(k(\theta) \nabla \theta)_{t}+\boldsymbol{b} \cdot \nabla \theta_{t}+c \theta_{t}=P\left(\sigma(\theta)|\nabla u|^{2}\right)_{t}$, where

$$
\nabla \cdot(k(\theta) \nabla \theta)_{t}=k^{\prime \prime}(\theta) \theta_{t}|\nabla \theta|^{2}+2 k^{\prime}(\theta) \nabla \theta_{t} \nabla \theta+k^{\prime}(\theta) \theta_{t} \Delta \theta+k(\theta) \Delta \theta_{t}
$$

and

$$
\begin{aligned}
& \left\|\left(\sigma(\theta)|\nabla u|^{2}\right)_{t}\right\|_{L^{2}(\Omega)} \\
\leq & \left\|\sigma^{\prime}(\theta) \theta_{t}|\nabla u|^{2}\right\|_{L^{2}(\Omega)}+2\left\|\sigma(\theta) \nabla u \nabla u_{t}\right\|_{L^{2}(\Omega)} \\
\leq & C\left(\left\|\theta_{t}\right\|_{L^{2}(\Omega)}\|u\|_{W^{1, \infty}(\Omega)}^{2}+\|u\|_{W^{1, \infty}(\Omega)}\left\|u_{t}\right\|_{W^{1,2}(\Omega)}\right) \\
\leq & C
\end{aligned}
$$


so it is easy to get the following estimates by (65),

$$
\left\|\theta_{t}(t)\right\|_{H^{2}(\Omega)} \leq C\left(1+\left\|\theta_{t t}(t)\right\|_{L^{2}(\Omega)}\right)
$$

In order to obtain estimate of $t\left\|\theta_{t t}(t)\right\|_{L^{2}(\Omega)}$, we differentiate (50) with respect to $t$ and let $\eta=\theta_{t t}$, similar disposal like before, and in view of (66) and (67), we get

$$
\left\|\theta_{t t}\right\|_{L^{2}(\Omega)}^{2}+\frac{d}{d t}\left\|\nabla \theta_{t}\right\|_{L^{2}(\Omega)}^{2} \leq C
$$

Hence, if multiplying by $t$ and integrating in $t$, it follows

$$
\int_{0}^{t} \tau\left\|\theta_{t t}\right\|_{L^{2}(\Omega)}^{2} d \tau+t\left\|\nabla \theta_{t}\right\|_{L^{2}(\Omega)}^{2} \leq C t+\int_{0}^{t}\left\|\nabla \theta_{t}\right\|_{L^{2}(\Omega)}^{2} d \tau \leq C
$$

by virtue of (62) and (64). We then differentiate (59) with respect to $t$ and let $\eta=\theta_{t t}$ to have

$$
\begin{aligned}
& \frac{1}{2} \frac{d}{d t}\left\|\theta_{t t}\right\|_{2}^{2}+\left((k(\theta) \nabla \theta)_{t t}, \theta_{t t}\right)+\left(\boldsymbol{b} \cdot \nabla \theta_{t t}, \theta_{t t}\right)+\left(c \theta_{t t}, \theta_{t t}\right) \\
= & \left((\sigma(\theta) u \nabla u)_{t t}, \theta_{t t}\right)+\left((f u)_{t t}, \theta_{t t}\right)
\end{aligned}
$$

where

$$
\begin{gathered}
(k(\theta) \nabla \theta)_{t t}=k^{\prime \prime}(\theta) \theta_{t}^{2} \nabla \theta+k^{\prime}(\theta) \theta_{t t} \nabla \theta+2 k^{\prime}(\theta) \theta_{t} \nabla \theta_{t}+k(\theta) \nabla \theta_{t t} \\
(f u)_{t t}=f_{t t} u+2 f_{t} u_{t}+f u_{t t}
\end{gathered}
$$

we could use similar method as above to treat them separately, then, if condition (15) is satisfied, we obtain

$$
\frac{d}{d t}\left\|\theta_{t t}\right\|_{L^{2}(\Omega)}^{2}+\alpha\left\|\nabla \theta_{t t}\right\|_{L^{2}(\Omega)}^{2} \leq C\left(\left\|(\sigma(\theta) u \nabla u)_{t t}\right\|_{L^{2}(\Omega)}^{2}+\left\|u_{t t}\right\|_{L^{2}(\Omega)}^{2}\right)
$$

Differentiating (57) with respect to $t$, we have

$$
\left(\sigma(\theta) \nabla u_{t t}, \nabla v\right)+\left(\sigma(\theta)_{t t} \nabla u+2 \sigma(\theta)_{t} \nabla u_{t}, \nabla v\right)=\left(f_{t t}, v\right) \quad \forall v \in H_{0}^{1}
$$

with $v=u_{t t}$, it follows

$$
\begin{aligned}
\left\|u_{t t}\right\|_{H^{1}(\Omega)} \leq & C\left(\left(\left\|\theta_{t t}\right\|_{L^{2}(\Omega)}+\left\|\theta_{t}\right\|_{L^{2}(\Omega)}\left\|\theta_{t}\right\|_{L^{\infty}(\Omega)}\right)\|u\|_{W^{1, \infty}(\Omega)}\right. \\
& \left.+\left\|\theta_{t}\right\|_{L^{\infty}(\Omega)}\left\|u_{t}\right\|_{W^{1,2}(\Omega)}+\left\|f_{t t}\right\|_{L^{2}(\Omega)}^{2}\right) \\
\leq & C\left(1+\left\|\theta_{t t}\right\|_{L^{2}(\Omega)}\right)
\end{aligned}
$$


For the term

$$
\begin{gathered}
(\sigma(\theta) u \nabla u)_{t t}=\sigma(\theta)_{t t} u \nabla u+\sigma(\theta) u_{t t} \nabla u \\
+\sigma(\theta) u \nabla u_{t t}+2 \sigma(\theta)_{t} u_{t} \nabla u+2 \sigma(\theta){ }_{t} u \nabla u_{t}+2 \sigma(\theta) u_{t} \nabla u_{t}
\end{gathered}
$$

we estimate similarly to [16] to get $\left\|(\sigma(\theta) u \nabla u)_{t t}\right\|_{L^{2}(\Omega)} \leq C\left(1+\left\|\theta_{t t}\right\|_{L^{2}(\Omega)}\right)$, together with (70) show (69) could be estimated by

$$
\frac{d}{d t}\left\|\theta_{t t}\right\|_{L^{2}(\Omega)}^{2}+\left\|\nabla \theta_{t t}\right\|_{L^{2}(\Omega)}^{2} \leq C\left(1+\left\|\theta_{t t}\right\|_{L^{2}(\Omega)}^{2}\right)
$$

If we multiply by $t^{2}$ and integrate, and in view of (68), it follows

$$
t^{2}\left\|\theta_{t t}(t)\right\|_{L^{2}(\Omega)}^{2}+\int_{0}^{t} \tau^{2}\left\|\nabla \theta_{t t}\right\|_{L^{2}(\Omega)}^{2} d \tau \leq C\left(1+\int_{0}^{t} \tau\left\|\theta_{t t}\right\|_{L^{2}(\Omega)}^{2} d \tau\right) \leq C
$$

which completes the proof.

\section{Blow-up result}

In this section we are interested to investigate under what condition the solution exists globally or finite time blow-up? We consider the problem as follows:

$$
\begin{cases}\text { (i) } \quad-\nabla \cdot(\sigma(\theta) \nabla u)=f & \text { in } \Omega \times(0, T) \\ \text { (ii) } \quad \theta_{t}-\nabla \cdot(k(\theta) \nabla \theta)+\boldsymbol{b} \cdot \nabla \theta+c \theta=\sigma(\theta)|\nabla u|^{2} & \text { in } \Omega \times(0, T) \\ \text { (iii) } \quad u=u_{0}, & x \in \Gamma, t>0 \\ \text { (iv) } \quad \partial \theta / \partial v=0, & x \in \Gamma, t>0 \\ \text { (v) } \quad \theta(x, 0)=\theta_{0}, & x \in \Omega\end{cases}
$$

where $\partial / \partial v$ is the outward normal derivative of $\partial \Omega$.

We note that our difficulty to treat problem (71) compared to general considered problem lies in the convection term $\boldsymbol{b} \cdot \nabla \theta$ and reaction term $c \theta$. From the physical point of view in [8], any solution $\theta$ to (71) can be written as $\theta(x, t)=\tilde{\theta}(x-t \boldsymbol{b}, t)$, by a variable transformation, we can obtain the 
equations that $\tilde{\theta}$ and corresponding solution $\tilde{u}$ satisfy,

$$
\begin{cases}\text { (i) } \quad-\nabla \cdot(\sigma(\tilde{\theta}) \nabla \tilde{u})=f & \text { in } \Omega_{t} \times\{t>0\} \\ \text { (ii) } \quad \tilde{\theta}_{t}-\nabla \cdot(k(\tilde{\theta}) \nabla \tilde{\theta})+c \tilde{\theta}=\sigma(\tilde{\theta})|\nabla \tilde{u}|^{2} & \text { in } \Omega_{t} \times\{t>0\} \\ \text { (iii) } \quad \tilde{u}=\tilde{u}_{0}=u_{0}(x-t \boldsymbol{b}, t), & x \in \Gamma, t>0 \\ \text { (iv) } \quad \partial \tilde{\theta} / \partial v=0, & x \in \Gamma, t>0 \\ \text { (v) } \quad \tilde{\theta}(x, 0)=\theta_{0}, & x \in \Omega_{t}\end{cases}
$$

where we still write $x, t$ if not causing any confusion and $\Omega_{t}=\Omega-t \boldsymbol{b}$. We note that this transformation does not change the shape of the boundary $\Gamma$ of $\Omega$ and initial value $\theta_{0}$. In this case we see that if $\tilde{\theta}$ blow up so does $\theta$ and vise versa, thus the convection term has no effect on whether solution is blow-up in finite time. So we turn our attention to problem (72). We assume that

$$
\begin{gathered}
\theta_{0} \geq 0, \quad x \in \Omega \\
0<k(s), \sigma(s)<\infty, \forall s \geq 0 ; \sigma(s) \text { differentiable, } \sigma^{\prime}(s) \geq 0, \forall s \geq 0
\end{gathered}
$$

and

$$
\int_{0}^{\infty} \frac{d s}{\sigma(s)}<\infty
$$

From [10], if $d \gamma(x)$ is the superficial measure on $\Gamma$, then

$$
\lambda \rightarrow \int_{\Gamma}|u-\lambda|^{2} d \gamma(x)
$$

achieves its minimum value for

$$
\lambda=\bar{u}=\frac{1}{|\Gamma|} \int_{\Gamma} u d \gamma(x)
$$

thus, if we set $u_{\Omega_{t}}=\frac{1}{\left|\Omega_{t}\right|} \int_{\Omega_{t}} u d x$, we have for some constant $C$,

$$
\begin{aligned}
\int_{\Gamma}|u-\bar{u}| d \gamma(x) & \leq \int_{\Gamma}\left|u-u_{\Omega_{t}}\right| d \gamma(x) \\
& \leq C \int_{\Omega_{t}}|\nabla u|^{2} d x \quad \forall u \in H^{1}\left(\Omega_{t}\right)
\end{aligned}
$$


so we get

$$
\int_{\Gamma}|u-\bar{u}|^{2} d \gamma(x) \leq C \int_{\Omega_{t}}|\nabla u|^{2} d x \quad \forall u \in H^{1}\left(\Omega_{t}\right)
$$

where $C$ denotes the best constant. Then we have the following result:

Theorem 4. Assume that $c$ is nonpositive function and

$$
\int_{\Omega} \int_{\theta_{0}}^{\infty} \frac{d s}{\sigma(s)} d x<\frac{1}{C} \int_{0}^{\infty} \int_{\Gamma}\left|\tilde{u}_{0}-\overline{\tilde{u}}_{0}\right|^{2} d \gamma(x) d t
$$

where

$$
\tilde{u}_{0}=u_{0}(x-t \boldsymbol{b}, t), \overline{\tilde{u}}_{0}=\frac{1}{|\Gamma|} \int_{\Gamma} \tilde{u}_{0} d \gamma(x)
$$

then problem (71) cannot have a smooth global solution.

Proof. The proof is similar to that of Theorem 5.1 in [10].

Remark 3. In one dimension case, when $k \equiv 1$ and $\boldsymbol{b}$ is a function of $x$ with $\frac{1}{2} \nabla \cdot \boldsymbol{b}+c \geq 0$, we could show that $\theta(x, t)$ blow up globally. In other words, if $t^{*}$ denotes the blowup time when

$$
\theta(x, t) \rightarrow \infty, \quad \text { a.e. } x \in \Omega \quad \text { when } t \rightarrow t^{*}
$$

Indeed, consider $\Omega=(0,1)$, then integrate the equation

$$
\left(\sigma(\theta) u^{\prime}\right)^{\prime}=f
$$

it follows

$$
u^{\prime}=\frac{\int_{0}^{1} f(x, t) d x+C(t)}{\sigma(\theta)}
$$

thus the equation satisfied by $\theta$ reads

$$
\theta_{t}-\theta_{x x}+\boldsymbol{b} \cdot \theta_{x}+c \theta=\frac{\left(\int_{0}^{1} f(x, t) d x+C(t)\right)^{2}}{\sigma(\theta)}
$$


Differentiating in $x$ and let $\eta=\theta_{x}$, we see that

$$
\eta_{t}-\eta_{x x}+\boldsymbol{b} \cdot \eta_{x}+(\nabla \cdot \boldsymbol{b}+c) \eta=-\frac{\sigma^{\prime}(\theta)\left(\int_{0}^{1} f(x, t) d x+C(t)\right)^{2}}{\sigma(\theta)^{2}} \eta
$$

with initial boundary

$$
\eta(x, t)=0, x=0,1 \quad \eta(x, 0)=\left(\theta_{0}\right)_{x}
$$

Assuming that $\left(\theta_{0}\right)_{x} \in L^{\infty}(0,1)$, and noting that

$$
\frac{\sigma^{\prime}(\theta)\left(\int_{0}^{1} f(x, t) d x+C(t)\right)^{2}}{\sigma(\theta)^{2}} \geq 0, \frac{1}{2} \nabla \cdot \boldsymbol{b}+c \geq 0
$$

then it follows from the parabolic maximum principle that

$$
\left|\theta_{x}\right|_{\infty} \leq\left|\left(\theta_{0}\right)_{x}\right|_{\infty}
$$

i.e.

$$
\theta(x, t)=\int_{x_{0}}^{x} \theta_{x}(x, t) d x+\theta\left(x_{0}, t\right)
$$

shows that if $\theta\left(x_{0}, t\right)$ blows up, then $\theta$ blows up for any $x$.

Remark 4. We note that the result of Theorem 4 is independent of $f$, then, without loss of generality, we specialize the problem (72) in one dimension with $f=0$ to show the sharpness of (76). Still consider $\Omega=(0,1), \tilde{\theta}(0)=$ $\theta_{0}=$ Const and look for a solution $\tilde{\theta}=\tilde{\theta}(t)$ depending on $t$ only. Set

$$
\tilde{u}_{0}(-t \boldsymbol{b}, t)=a_{0}(t), \quad \tilde{u}_{0}(1-t \boldsymbol{b}, t)=a_{1}(t)
$$

then the equation satisfied by $u$ leads to

$$
\tilde{u}(x, t)=a_{0}(t)+x\left(a_{1}(t)-a_{0}(t)\right)
$$

thus, the equation satisfied by $\theta$ becomes

$$
\tilde{\theta}_{t}-c \tilde{\theta}=\sigma(\tilde{\theta})\left(a_{1}(t)-a_{0}(t)\right)^{2}
$$


i.e.

$$
\int_{0}^{t} \frac{\tilde{\theta}_{t}+c \tilde{\theta}}{\sigma(\tilde{\theta})} d t=\int_{0}^{t}\left(a_{1}(t)-a_{0}(t)\right)^{2} d t
$$

or

$$
\int_{\theta_{0}}^{\tilde{\theta}} \frac{d s}{\sigma(s)}+\int_{0}^{t} \frac{c \tilde{\theta}}{\sigma(\tilde{\theta})} d s=\int_{0}^{t}\left(a_{1}(s)-a_{0}(s)\right)^{2} d s
$$

In this case, the failure of (76) reads

$$
\int_{\theta_{0}}^{\infty} \frac{d s}{\sigma(s)} \geq \int_{0}^{\infty}\left(a_{1}(s)-a_{0}(s)\right)^{2} d s
$$

which implies that (72) has a global solution which is bounded when

$$
\int_{\theta_{0}}^{\infty} \frac{d s}{\sigma(s)} \geq \int_{0}^{\infty}\left(a_{1}(s)-a_{0}(s)\right)^{2} d s
$$

and is unbounded otherwise.

Acknowledgements. The authors would like to thank Professor Hongwei Wu from Southeast University, Mr. Zhifen Xu and Miss Danni Hu from Nanjing University of Information Science \& Technology for their helpful suggestions and discussions.

\section{REFERENCES}

[1] P. Avenas, J.F. Agassant and J.Ph. Sergent, La mise en forme des matières plastiques. Lavoisier (1982).

[2] J. Baranger and A. Mikelić, Stationary solutions to a quasi-Newtonian flow with viscous heating. Math. Models Methods Appl. Sci., 5 (1995), 725-738.

[3] J. Bass, Thermoelasticity. McGraw-Hill Encyclopedia of Physics, S.P. Parker ed., McGraw-Hill, New York (1982).

[4] S. Wardi, A convergence result for an iterative method for the equations of a stationary quasi-newtonian flow with temperature dependent viscosity. M2AN: Modél. Math. Anal. Numér., 32 (1998), 391-404.

[5] J. Zhu, A.F.D. Loula, J. Karam F. and J.N.C Guerreiro, Finite element analysis of a coupled thermally dependent viscosity flow problem. Comput. Appl. Math., 26 (2007), 45-66. 
[6] R. Lewandowski, Analyse Mathématique et Océanographie. Masson (1997).

[7] B. Mohammadi and O. Pironneau, Analysis of the k-Epsilon model. Masson, (1994).

[8] J. Aguirre and M. Escobedo, On the blow-up of solutions of a convective reaction diffusion equation. Proceedings of Royal Society of Edinburgh, 123A (1993), $433-460$.

[9] W. Allegretto and H. Xie, Solutions for microsensor thermistor equations in the small bias case. Proc. Roy. Soc. Edingburgh Ser. A, 123 (1993), 987-999.

[10] S.N. Antontsev and M. Chipot, The thermistor problem: existence, smoothness, uniqueness, blowup. SIAM, J. Math. Anal., 25 (1992), 1128-1156.

[11] A. Bossavit and J.-F. Rodrigues, On the electromagnetic "induction heating" problem in bounded domains. Adv. Math. Sci. Appl., 4 (1994), 79-92.

[12] X. Chen and A. Friedman, A free boundary problem for a nonlinear degenerate elliptic system modeling a thermistor. Ann. Scuola Norm. Sup. Pisa Cl. Sci., 19 (1992), 615-636.

[13] G. Cimatti, A bound for the temperature in the thermistor problem. IMA J. Appl. Math., 40 (1988), 15-22.

[14] G. Cimatti and G. Prodi, Existence results for a nonlinear elliptic system modelling a temperature dependent electrical resistor. Ann. Mat. Pura Appl., 152 (1989), $227-236$.

[15] S. Clain, J. Rappaz, M. Swierkosz and R. Touzani, Numerical modelling of induction heating for two-dimensional geometries. Math. Models Methods Appl. Sci., 3 (1993), 805-822.

[16] C.M. Elliott and S. Larsson, A finite element model for the time-dependent Joule heating problem. Math. Comp., 64 (1995), 1433-1453.

[17] J. Fan and $\mathrm{H}$. Wu, On the $N$-dimensional stationary drift-diffusion semiconductor equations. Nonlinear Anal., 43 (2001), 127-135.

[18] A. Friedman, Partial Differential Equations of Parabolic Type. Englewood Cliffs, N.J., Prentice-Hall (1964).

[19] T. Gallouët and R. Herbin, Existence of a solution to a coupled elliptic system. Appl. Math. Lett., 7 (1994), 49-55.

[20] D. Gilbarg and N.S. Trudinger, Elliptic Partial Differential Equations of Second Order, second edition, Springer-Verlag, Berlin (1983). 
[21] K. Gröger, $W^{1, p}$-estimates of solutions to evoluton equations corresponding to nonsmooth second order elliptic differential operators. Nonlinear Anal. Theory, Meth. \& Appl., 18 (1992), 569-577.

[22] P. Guan, Existence, boundedness and uniqueness of weak solution for the thermistor problem with mixed boundary conditions. Acta Mathematica Scientia, 18 (1998), 326-332.

[23] H.D. Howison and J.F. Rodrigues and M. Shillor, Stationary solutions to the thermistor problem. J. Math. Anal. Appl., 174 (1993), 573-588.

[24] A.F.D. Loula and J. Zhu, Finite element analysis of a coupled nonlinear system. Comput. Appl. Math., 20 (2001), 321-339.

[25] A. Quarteroni and A. Valli, Numerical Approximation of Partial Differential Equations. Springer Series in Computational Mathematics, Vol. 23, SpringerVerlag (1997).

[26] P. Shi, M. Shillor and X. Xu, Existence of a solution to the Stefan problem with Joule's heating. J. Differential Eq., 105 (1993), 239-263.

[27] Y. Wang, Nonlinear Partial Differential Equations. Southeast University Press, (1992).

[28] J. Xing and Y. Wang, The initial boundary value problem of semiconductor equations wiht heat conduction. Acta Math. Appl. Sinica (in Chinese), 21 (1998), 215-223.

[29] X. Xu, On the existence of bounded temperature in the thermistor problem with degeneracy. Nonlinear Anal., 42 (2000), 199-213.

[30] H.M. Yin, The semiconductor systems with temperature effect. J. Math. Anal. Appl., 196 (1995), 135-152.

[31] G. Yuan and Z. Liu, Existence and uniqueness of the $C^{\alpha}$-solution for the thermistor problem with mixed boundary values. SIAM J. Math. Anal., 25 (1994), $1157-1166$.

[32] J. Zhu and A.F.D. Loula, Mixed finite element analysis of a thermally nonlinear coupled problem. Numer. Methods Partial Differential Eq., 22 (2006), 180-196.

[33] $\mathrm{H}$. Wang, G. Li, J. Zhu and D. Hu, Existence and regularity of solution to a thermally coupled nonstationary sysytem. Chin. Quart. J. of Math., 23(4) (2008), 512-524.

[34] C.G. Simader, On Dirichlet's Boundary Value Problem. Lecture Notes in Mathematics 268, Springer-verlag, Berlin (1972). 